\title{
Antena de Parche Cortocircuitado con Polarización Circular y Sentido de Giro Reconfigurable para Aplicaciones en Satélites de Reducido Tamaño
}

\author{
Carlos A. Suárez-Fajardo*, Cristiam D. Martín-Jackson y Gustavo A. Puerto-Leguizamón \\ Universidad Distrital "Francisco José de Caldas", Facultad de Ingeniería, Grupo de investigación: \\ Radiación Electromagnética y Comunicaciones Ópticas (GRECO-LIMER), Bogotá-Colombia. \\ (e-mail: csuarezf@udistrital.edu.co; cdmartinj@correo.udistrital.edu.co; gapuerto@udistrital.edu.co) \\ ${ }^{*}$ Autor a quien debe ser dirigida la correspondencia.
}

Recibido Abr. 3, 2017; Aceptado Jun. 12, 2017; Versión final Jul. 8, 2017, Publicado Dic. 2017

\section{Resumen}

Este artículo presenta el diseño y caracterización de una antena microstrip de parche circular cortocircuitado, alimentada mediante un acoplador híbrido de cuadratura para lograr polarización circular reconfigurable. El elemento radiante posee un radio de $20.3 \mathrm{~mm}$ y las dimensiones totales de la antena son de $76 \mathrm{~mm} \times 76 \mathrm{~mm} \times 2.03 \mathrm{~mm}$, adecuadas para adosarla a una de las caras de un satélite (CubeSat $1 \mathrm{U}$ ). Los resultados de las medidas del prototipo muestran una ganancia máxima de $G_{0}=6,04 d B i$ y ancho de banda de relación axial de $48.56 \mathrm{MHz}(2 \%)$ respecto a $2 \mathrm{~dB}$ a la frecuencia central de $f_{0}=2,44 \mathrm{GHz}$; un ancho de banda de impedancia del $27.44 \%$ respecto a una magnitud del coeficiente de reflexión de $S_{11}<$ $-10 \mathrm{~dB}$, en el rango de $2,295 \mathrm{GHz}$ a $3,025 \mathrm{GHz}$ y frecuencia central de $f_{0}=2,44 \mathrm{GHz}$.

\section{Short-Circuited Patch Antenna with Circular Polarization and Reconfigurable Sense of Rotation for Small-Sized Satellite Application}

\begin{abstract}
This paper presents the design and characterization of a circular microstrip patch antenna, fed by a hybrid quadrature coupler to achieve reconfigurable circular polarization. The radiating element has a radius of $20.3 \mathrm{~mm}$ and the overall dimensions of the antenna are $76 \mathrm{~mm} \times 76 \mathrm{~mm} \times 2.03 \mathrm{~mm}$, suitable to attaching it to one of the faces of a satellite (CubeSat $1 U$ ). The measurement results of the prototype show a maximum gain of $G_{0}=6,04 \mathrm{dBi}$ and axial ratio bandwidth of $48.56 \mathrm{MHz}(2 \%)$ respect to $2 \mathrm{~dB}$ at the center frequency of $f_{0}=2,44 \mathrm{GHz}$; an impedance bandwidth of $27.44 \%$ respect to the magnitude of the reflection coefficient of $S_{11}<-10 \mathrm{~dB}$, in the range of $2,295 \mathrm{GHz}$ to $3,025 \mathrm{GHz}$ at the center frequency of $f_{0}=2,44 \mathrm{GHz}$.
\end{abstract}

Keywords: shorted circular patch antenna; circular polarization; reconfigurable antenna, satellite application 


\section{INTRODUCCIÓN}

En las últimas décadas, las antenas con polarización circular han sido integradas en sistemas de comunicaciones inalámbricas debido a que permiten combatir diversos fenómenos de propagación tales como: desvanecimiento por trayecto múltiple (Counselman, 1999), el efecto de rotación de Faraday en la ionosfera (Brookner, 1985), al igual que una reducción de las pérdidas por errores de orientación entre las antenas receptora y transmisora (Gao et al., 2014). Dentro de los sistemas inalámbricos que las usan se pueden mencionar los siguientes: comunicaciones por satélite, sistemas globales de navegación por satélite (GNSS), comunicaciones móviles, sensores inalámbricos, identificación por radiofrecuencia (RFID), transmisión de energía inalámbrica, redes inalámbricas de área local (WLAN) y personal (WPAN), interoperabilidad mundial para acceso a microondas (WiMAX) y sistemas de radiodifusión directa de televisión (DBS).

Las antenas de microcinta brindan una solución efectiva de fácil fabricación, bajo peso, reducido costo, bajo perfil y fácil adaptación en superficies curvas, por tanto, es una de las tecnologías preferidas por los investigadores a la hora de diseñar antenas con polarización circular. Por otra parte, satisfacer los requerimientos en esta tecnología que garanticen la polarización circular en cuanto a la excitación de dos modos ortogonales con una diferencia de fase de $90^{\circ}$ entre ellos, puede ser logrado de forma simple mediante el uso de acopladores híbridos en cuadratura. Así mismo, son de fácil aplicación las otras técnicas como es el caso de ajustar las dimensiones físicas del parche o mediante el uso de uno, dos, o más alimentadores. De igual manera, se han empleado técnicas como acoplamiento por ranura, alimentadores acoplados electromagnéticamente y guías de ondas coplanares (CPW), (Gao et al., 2014; Pozar, 1995). Algunos autores han propuesto técnicas integrales que permiten diseñar antenas de parche de tamaño reducido, incluyendo una mejora en su relación axial y de adaptación de los puertos y una notable reducción de las ondas de superficie para parches con geometría circular de radio externo determinado (Jackson et al., 1993).

Las antenas para satelitales en estándar CubeSat requieren de diseños de tamaño reducido y altas prestaciones en parámetros como anchos de banda de relación axial e impedancia y diagramas de radiación directivos con ángulo útil apropiado. A este respecto, a continuación de exploran diversas estrategias para lograr estos objetivos. En Cheng et al. (2014) se propone el diseño de un parche cuadrado con polarización circular a derechas (RHCP) mediante plano de masa de $70 \mathrm{mmX} 100 \mathrm{~mm}$, en el cual se practican ranuras para reducir el tamaño, la diferencia de fase de $90^{\circ}$ se logra mediante un divisor de potencia Wilkinson y una línea de desfase de $\lambda / 4$, la antena presenta un ancho de banda de impedancia del $7 \%$ y una ganancia máxima de $7 \mathrm{dBi}$ a la frecuencia de $2,45 \mathrm{GHz}$. Por otra parte, Jie et al. (2016) presentan el diseño de una antena de parche circular con dos ranuras asimétricas circulares para generar dos modos ortogonales y de esta forma generar polarización circular, este parche se excita mediante una línea de transmisión acoplada por proximidad, la antena tiene dimensiones de $60 \mathrm{~mm} \times 60 \mathrm{~mm} \times 3,2 \mathrm{~mm}$ y logra un pico de ganancia de $6,1 \mathrm{dBi}$ para la frecuencia de $2,39 \mathrm{GHz}$ y un ancho de banda de $5,77 \%$ con frecuencia central en 2,37GHz. En este mismo sentido, Berg et al. (2016) proponen una antena para aplicaciones en sistemas de posicionamiento global (GPS) con polarización circular a izquierdas y derechas, la cual hace uso de un plano de masa $100 \mathrm{~mm} \times 100 \mathrm{~mm}$, la antena consta de un parche cuadrado excitado mediante acople magnético por ranuras en el plano de masa y un acoplador híbrido en cuadratura como red de excitación para lograr polarización circular, el ancho de banda obtenido para la frecuencia de $1,575 \mathrm{GHz}$ es de $24 \mathrm{MHz}$ y la máxima ganancia obtenida para la misma frecuencia es de $5,2 \mathrm{dBi}$.

En Suárez et al. (2016a) proponen el diseño de una antena de parche en anillo circular cortocircuitado con polarización circular a izquierdas (LHCP) para aplicaciones en satélites CubeSat 1U, en la cual se logra un mejoramiento de la relación axial mediante el uso de hendiduras (slits) y prolongaciones (stubs), mediante lo cual se obtiene un ancho de banda de impedancia del 3,32 \% en el rango de $2,37 \mathrm{GHz}$ a $2,45 \mathrm{GHz}$ para un coeficiente de reflexión inferior a $-10 \mathrm{~dB}$ y una ganancia máxima de $6,2 \mathrm{dBi}$ a una frecuencia central de 2,41GHz; por otro lado, en (Suárez et al. 2016b), se presenta el diseño de una antena tipo parche en anillo circular cortocircuitado, con polarización circular y sentido de giro reconfigurable para aplicaciones en satélites CubeSat $1 \mathrm{U}$, los resultados muestran un ancho de banda de impedancia del $33,33 \%$ en el rango de $1,9 \mathrm{GHz}$ a $2,66 \mathrm{GHz}$ para un coeficiente de reflexión inferior a $-10 \mathrm{~dB}$ y una ganancia máxima de $6.1 \mathrm{dBi}$ a una frecuencia central de 2,35GHz; así mismo, en (Suárez et al 2016c) se presenta el desarrollo de una antena con polarización circular y doble resonancia para aplicaciones en sistemas GPS (Global Position Systems), la cual hace uso de dos parches rectangulares apilados y separados por aire, logrando un ancho de banda de impedancia $18,03 \mathrm{MHz}$ para la frecuencia de $1,227 \mathrm{GHz}$ y $17,66 \mathrm{MHz}$ para la frecuencia central de $1,575 \mathrm{GHz}$, con ganancias de $5,4 \mathrm{dBi}$ y $6,8 \mathrm{dBi}$ respectivamente.

Por otra parte, en (Ernest et al, 2012) se presentan las simulaciones de una antena del tipo espiralogarítmica con geometría cónica para uso en satélites $3 U(10 \mathrm{~cm} \times 30 \mathrm{~cm})$, junto con el diseño del circuito 
simetrizador (BALUM). El ancho de banda de impedancia obtenido fue de $2 \mathrm{GHz}$ a $3.44 \mathrm{GHz}$, con directividades de $3 \mathrm{dBi}$ (banda baja) y $11 \mathrm{dBi}$ (banda alta). La antena requiere de un sistema de despliegue y no presenta los datos de ganancia ni relación axial. Por otra parte, en (Wang, et al, 2012) se reportan las simulaciones de una antena de banda ancha con sustratos apilados y excitador con geometría en "L", en la cual se logra ancho de banda de impedancia del $98.4 \%$ en el rango de $3.3 \mathrm{GHz}$ a $9.5 \mathrm{GHz}$ y ganancia promedio de $6 \mathrm{dBi}$, sin embargo, la polarización es lineal y el perfil total es de $9.6 \mathrm{~mm}$. En este mismo sentido, en (Rani, et al, 2014) se presentan las simulaciones de una antena de parche con doble resonancia y polarización lineal, con ancho de banda de impedancia de $3.1 \mathrm{GHz}$ a $6.2 \mathrm{GHz}$ y de $7 \mathrm{GHz}$ a $10 \mathrm{GHz}$, sin embargo no reporta datos sobre ganancia y diagrama y su tamaño total es de $30 \mathrm{~mm} \times 40 \mathrm{~mm} \times 1.6 \mathrm{~mm}$. Finalmente, en (Shackelford, et al, 2003) se presenta el diseño y validación de dos tipos de antenas a saber: ranuras en "U" y excitadores con geometría en "L" y estudia diversas estrategias para reducir su tamaño, logrando una reducción del $94 \%$ con ancho de banda no inferior al $20 \%$ y ganancias en el intervalo entre $2 \mathrm{dBi}$ y $6,5 \mathrm{dBi}$; la polarización es lineal y el perfil es de $7 \mathrm{~mm}$ en el mejor de los casos.

En este artículo se presenta el diseño de una antena con polarización circular y sentido de giro configurable entre RHCP y LHCP, para ser adosada a una de las caras de un satélite $1 \mathrm{U}$ (cubo de 10cm de lado) en el espacio disponible para tal fin de $76 \mathrm{~mm} \times 76 \mathrm{~mm} \times 3,0 \mathrm{~mm}$ (descontando mecanismos de soporte). La propuesta hace uso de un parche circular cortocircuitado en el centro y excitación dual mediante acoplador hibrido de $90^{\circ}$; no requiere de mecanismo de despliegue y cumple con las especificaciones eléctricas deseables para el sistema de comunicaciones en el satélite. La antena diseñada posee dimensiones totales de $76 \mathrm{~mm} \times 76 \mathrm{~mm} \times 2,03 \mathrm{~mm}$, con un parche radiante circular con radio de $20,3 \mathrm{~mm}$, mediante lo cual se logra una ganancia máxima de $6,04 \mathrm{dBi}$ a una frecuencia de $2,44 \mathrm{GHz}$; un ancho de banda de relación axial de $48.56 \mathrm{MHz}(2 \%)$ y un ancho de banda de impedancia del $27,39 \%$ en el rango de $2,295 \mathrm{GHz}$ a $3,025 \mathrm{GHz}$ ambos para la misma frecuencia central de $2.44 \mathrm{GHz}$.

\section{METODOLOGÍA}

La Fig. 1 muestra las vistas lateral y frontal Fig. 1 (a) y (b) respectivamente para la geometría del parche circular cortocircuitado en el centro con alimentación dual propuesto en este artículo. El diseño permite la puesta a punto del parche y del acoplador híbrido de $90^{\circ}$ por separado, aprovechando el hecho que se unen los dos planos de masa de estos elementos como se muestra en la Fig. 1 (a), evitando así la incidencia en el funcionamiento del uno sobre el otro. El corto circuito en el centro reduce la energía de los modos de orden superior, por otra parte, es menos crítico adaptar el puerto a $50 \Omega$ debido a que el disco posee una impedancia entre $0 \Omega$ y $100 \Omega$ (borde), al tiempo que se reduce la energía almacenada en el parche disminuyendo el factor de calidad y aumentando el ancho de banda respecto a un disco convencional.

El prototipo construido con base en la geometría diseñada será caracterizado en impedancia con el analizador vectorial de redes (VNA) Rohde\&Schwartz ZVL13. La ganancia se caracterizará usando el método de ganancia absoluta con tres antenas y la relación axial mediante un dipolo que gira sobre el eje de propagación de la onda, ambas medidas en cámara anecoica (Balanis, 2005).

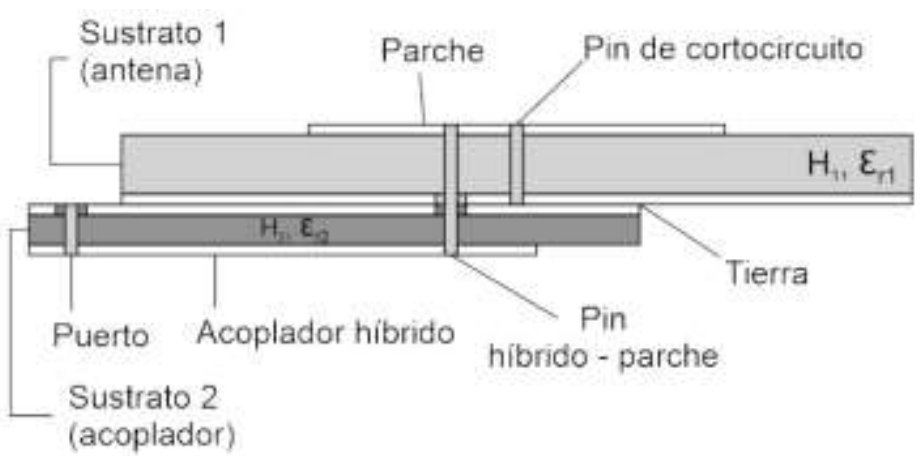

(a)

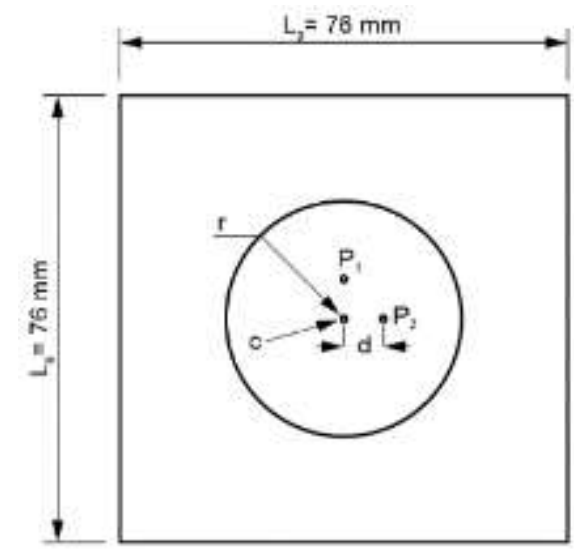

(b)

Fig. 1: (a) Vista lateral de la antena y la red de excitación. (b) Vista frontal de la antena

\section{Diseño del elemento radiante}

En esta sección se presenta el proceso de diseño del elemento radiante, para lo cual se parte de la selección del primer sustrato (ubicado en la parte superior en la Fig. 1 (a)) con las siguientes especificaciones de constante dieléctrica, tangente de pérdidas y espesor: $\varepsilon_{r 1}=3, \tan _{1}=0,0014, y H_{1}=$ 
1,524mm respectivamente, para posteriormente hacer uso de la ecuación (1) relacionada a continuación, mediante las cuales se determina el radio efectivo $(r)$ del parche el cual tiene en cuenta el efecto del campo en el borde de la antena (fringing) que la hace lucir eléctricamente más larga (Balanis, 2005).

$$
r=\frac{8,791 \times 10^{9}}{f_{r} \sqrt{\epsilon_{r}\left(1+\frac{2 h f_{r}}{8,791 \times 10^{9} \pi \sqrt{\epsilon_{r}}}\left(\ln \left(\frac{8,791 \times 10^{9} \pi}{2 h f_{r} \sqrt{\epsilon_{r}}}\right)+1,7726\right)\right)}}
$$

En esta ecuación, $h$ es el espesor del sustrato en $\mathrm{cm}$, que para el caso de la antena es $h=H_{1}$.

Posteriormente se lleva a cabo un proceso de puesta a punto de parámetros mediante el programa de análisis electromagnético "Ansoft High Frequency Structure Simulator (HFSS)", que integra herramientas profesionales para el diseño de antenas y permite adelantar un análisis paramétrico de la estructura en no más de 5 minutos. Para este propósito se cuenta con dos grados de libertad a saber: radio del parche (notado como r en la Fig. 1(a)) y posición de los excitadores (notados como d en la misma Figura).

Mediante la ecuación (1), se determina el radio efectivo de la antena para una frecuencia de resonancia de $f_{r}=2,45 \mathrm{GHz}$ y las especificaciones del sustrato 1 notadas antes, con lo cual se obtiene un radio efectivo del parche circular de $r=20,72 \mathrm{~mm}$; fijando la longitud del lado del plano de masa cuadrado en $\mathrm{L}_{\mathrm{g}}=76 \mathrm{~mm}$ (por espacio disponible). La Fig. 1(b) muestra la geometría de la antena en la cual se notan tres puntos a saber: los puertos de alimentación $\left(P_{1}\right.$ y $\left.P_{2}\right)$ que forman un ángulo de $90^{\circ}$ entre sí, separados una distancia $d$ del centro del parche y el punto central $(c)$ que denota el corto que une el parche con el plano de masa. El sustrato 1 (Fig. 1a) se selecciona con mayor constante dieléctrica que el sustrato 2 con el fin de reducir el tamaño del parche y el sustrato 2 posee menor espesor para disminuir efectos inductivos de los excitadores.

Con base en las dimensiones antes determinadas $\left(r, L_{g}\right)$, se lleva a cabo una serie de análisis paramétricos con HFSS, mediante las cuales se pretende mejorar el comportamiento de la antena base inicial en cuanto a parámetros como: adaptación de los puertos $\left(S_{11} y S_{22}\right)$, acoplamiento entre los mismos $\left(S_{12}\right.$ y $\left.S_{21}\right)$, ganancia y relación axial. La Fig. 2(a) muestra los resultados de las simulaciones del efecto generado por el cambio de la dimensión del radio del parche $(r)$, partiendo de un radio de $r=20,72 \mathrm{~mm}$ y separación $d=$ $6 \mathrm{~mm}$ (antena base inicial); en los parámetros de adaptación $\left(S_{11}\right)$ y de acoplamiento entre puertos $\left(S_{12}\right)$. Los resultados del comportamientos de los parámetros $\left(S_{22}\right.$ y $\left.S_{21}\right)$ son similares a los anteriores por lo tanto no se muestran en la figura. En este mismo sentido, la Fig. 2(b) muestra los resultados de las simulaciones de la ganancia y la relación axial para estos mismos valores del radio $r$ y separación entre los puertos respecto al centro $(d=6 \mathrm{~mm})$.

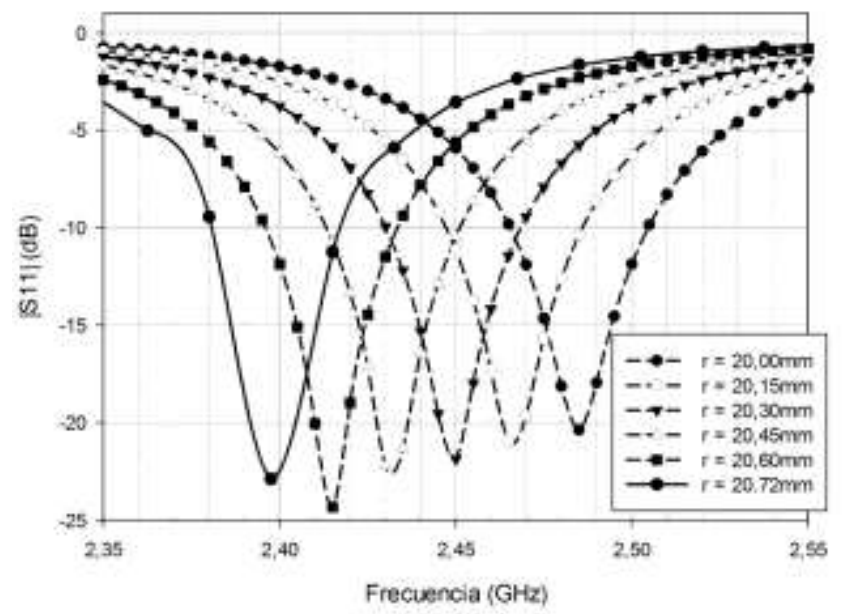

(a)

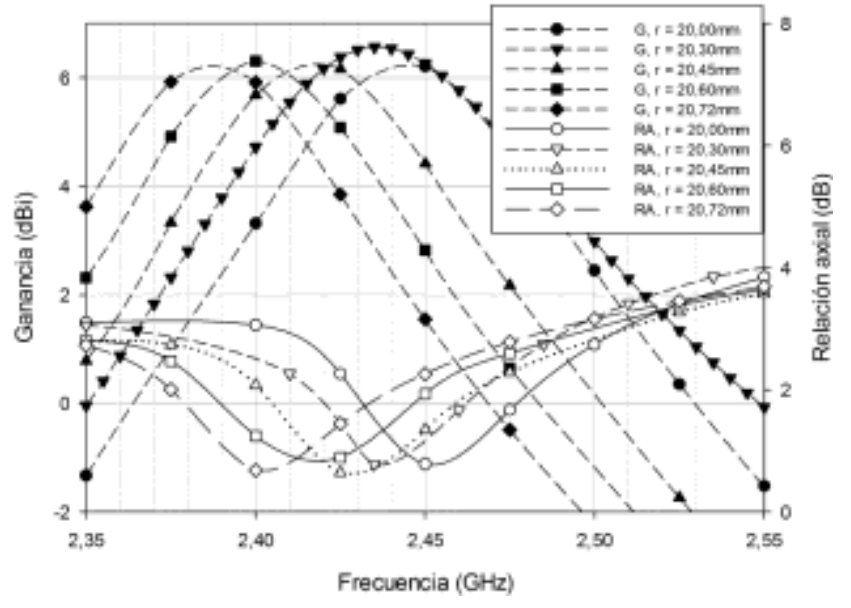

(b)

Fig. 2: (a) Comportamiento de los parámetros $\mathrm{S}_{11} \mathrm{y} \mathrm{S}_{12}$ para un radio ( $\mathrm{r}$ ) en el intervalo $20 \mathrm{~mm} \leq \mathrm{r} \leq 20,72 \mathrm{~mm}$. (b). Comportamiento de la ganancia y relación axial para un radio en el mismo intervalo.

En la Fig. 3 se presentan las simulaciones del comportamiento de la magnitud del coeficiente de reflexión del puerto $1\left(S_{11}\right)$ en función de la distancia $d$, en el rango de $3 \mathrm{~mm} \leq d \leq 8 \mathrm{~mm}$, manteniendo un radio constante de $r=20,3 \mathrm{~mm}$. De estas simulaciones se extrae que la mejor adaptación del puerto ocurre cuando $d=6 \mathrm{~mm}$. El parámetro $\left(S_{22}\right)$ se comporta de igual forma, por tanto no se incluye en esta figura. 


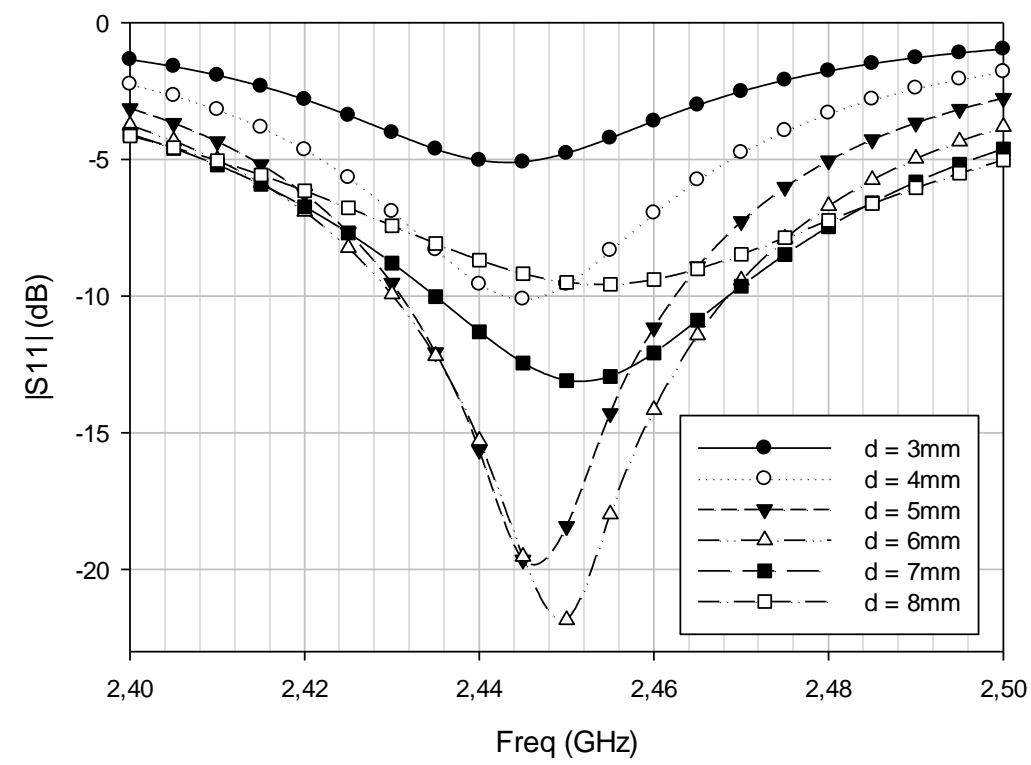

Fig. 3: Adaptación Vs. posición de excitadores

\section{Diseño del acoplador híbrido en cuadratura}

El diseño de este dispositivo se basa en el planteamiento expuesto en (Pozar D. M, 2005) y sus parámetros geométricos se muestran en la Fig. 4. La geometría inicial parte del modelo teórico para luego adelantar un proceso de puesta a punto mediante el simulador electromagnético "Ansoft Designer", el cual posee herramientas robustas para diseñar este tipo de dispositivos. El sustrato seleccionado para el acoplador posee las especificaciones de constante dieléctrica y tangente de pérdidas de: $\varepsilon_{r}=2,17, \tan \delta=0,0009$ un espesor de $H_{2}=0,508 \mathrm{~mm}$ y un plano de masa de dimensiones $L_{H}=50 \mathrm{~mm}$ y $A_{H}=35 \mathrm{~mm}$ resultando del proceso de puesta a punto las siguientes dimensiones: $L_{1}=20,20 \mathrm{~mm}, L_{2}=21,28 \mathrm{~mm}, W_{1}=1,89 \mathrm{~mm}, W_{2}=$ $W_{3}=1,1 \mathrm{~mm}$ (Fig. 4).

En la Fig. 5 se presentan los gráficos de la magnitud del coeficiente de reflexión $\left(S_{11}\right)$ para el puerto 1 de entrada del híbrido $\left(P_{1 H}\right)$ y el parámetro de transmisión entre los puertos $P_{1 H}$ y $P_{2 H}\left(S_{12}\right)$. Los resultados de la simulación muestran un ancho de banda de $33,73 \%$ con referencia de $-10 \mathrm{~dB}$ en el intervalo de $2,07 \mathrm{GHz}$ a $2,91 \mathrm{GHz}$, así mismo, la diferencia de fase de $90^{\circ}$ requerida para obtener polarización circular se mantiene en el rango de $90^{\circ} \pm 1^{\circ}$ en el intervalo de frecuencias desde $2,3 \mathrm{GHz}$ hasta $2,78 \mathrm{GHz}$.

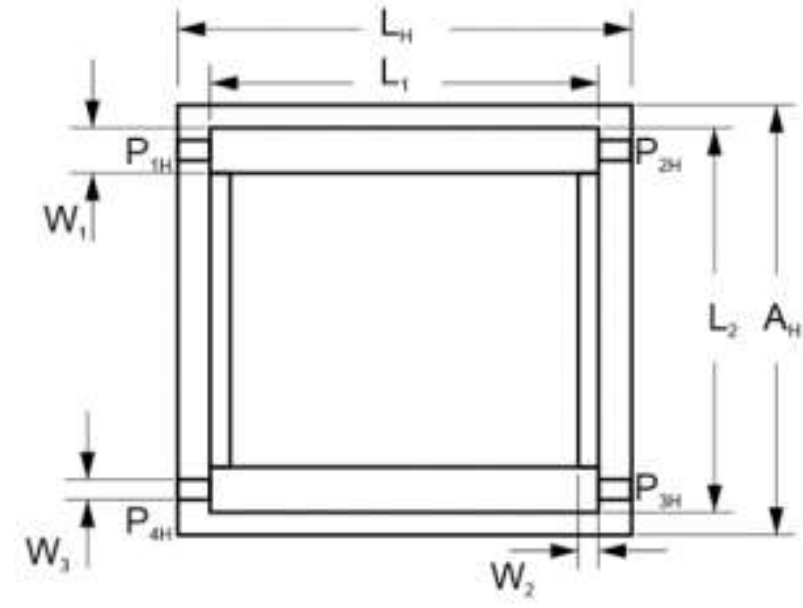

Fig.4: Cotas para el acoplador híbrido

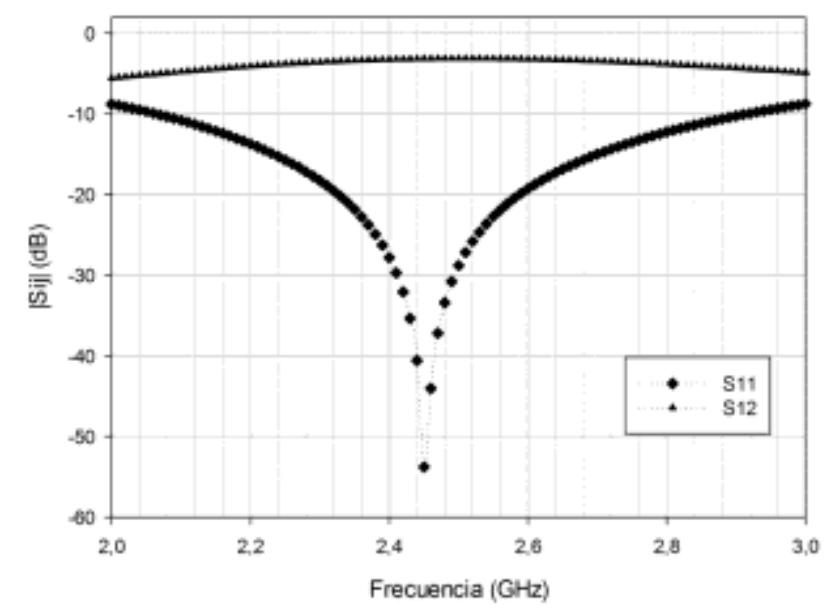

Fig. 5: Parámetros de adaptación $\left(S_{11}\right)$ y transmisión $\left(S_{12}\right)$ del acoplador híbrido.

La antena final integra el parche optimizado (Fig. 1(b)), con dimensiones: $r=20,3 \mathrm{~mm}, d=6 \mathrm{~mm}$, plano de masa cuadrado de lado $\mathrm{L}_{\mathrm{g}}=76 \mathrm{~mm}$, diseñado sobre el sustrato 1 (Fig. $1(\mathrm{a})$ ), con especificaciones $\varepsilon_{r 1}=$ $3, \tan \delta_{1}=0,0014, y H_{1}=1,524 \mathrm{~mm}$. Así mismo, el acoplador híbrido de $90^{\circ}$ optimizado (Fig. 4) cuenta con las dimensiones: $L_{1}=20,20 \mathrm{~mm}, L_{2}=21,28 \mathrm{~mm}, W_{1}=1,89 \mathrm{~mm}, W_{2}=W_{3}=1,1 \mathrm{~mm}$. diseñado sobre el sustrato 2 con dimensiones $L_{H}=50 \mathrm{~mm}$ y $A_{H}=35 \mathrm{~mm}$ y especificaciones: $\varepsilon_{r}=2,17, \tan \delta=0,0009$ y un espesor de $\mathrm{H}_{2}=0,508 \mathrm{~mm}$. La integración de la antena y acoplador se lleva a cabo uniendo los puertos $P_{2 H}$ 
del híbrido con el puerto $P_{2}$ de la antena, de igual manera se une el puerto $P_{3 H}$ con el puerto $P_{1}$. Por otra parte, los planos de masa de antena e híbrido se unen y los conectores SMA se ubican en el sustrato 2 del hibrido en los puertos de entrada del mismo $\left(P_{1 H}, P_{4 H}\right)$.

\section{RESULTADOS}

En la Fig. 6 se observa una foto de las vistas superior e inferior del conjunto integrado de la antena con el acoplador híbrido, incluyendo los conectores SMA. Cabe notar que el sentido de giro depende del puerto seleccionado como entrada, cargando el puerto libre con una impedancia de $50 \Omega$.

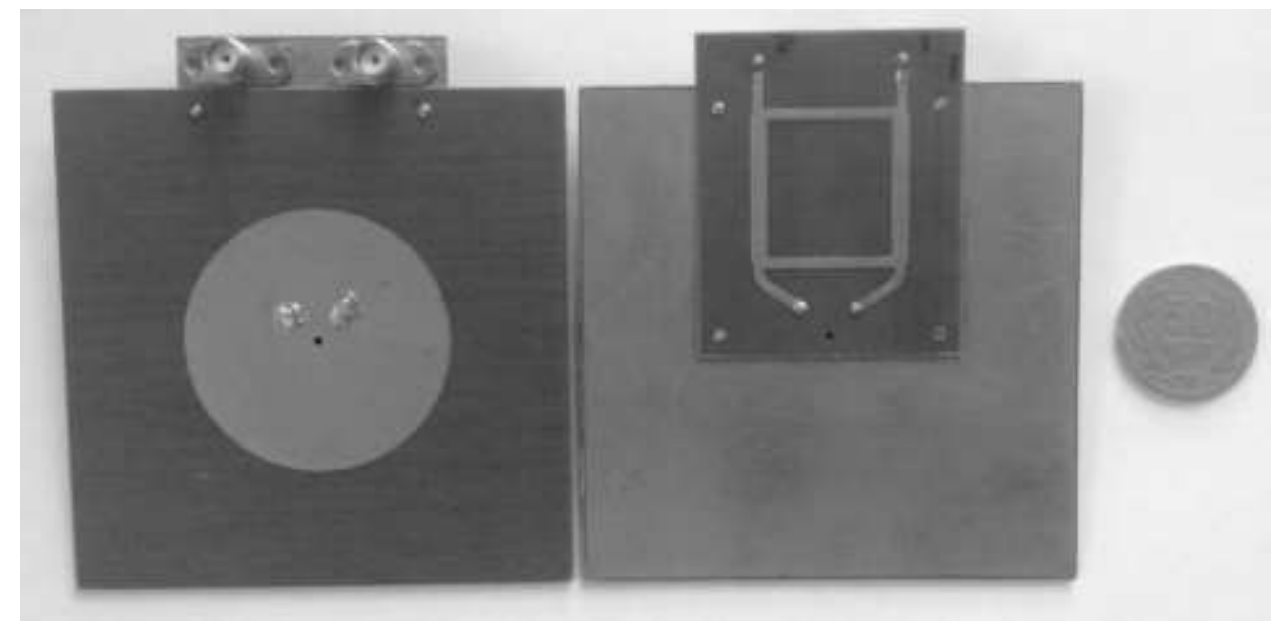

Fig. 6: Vista frontal de prototipo de antena circular cortocircuitada

El prototipo construido se caracteriza en adaptación y acople mediante el analizador vectorial de redes (VNA) Rohde\&Schwartz ZVL13. La Fig. 7(a) muestra la comparación entre los resultados de las medidas frente a las simulaciones del parámetro $S_{11}$. El resultado de las medidas muestran un ancho de banda de $730 \mathrm{MHz}(27,44 \%)$ para una frecuencia central de $f_{0}=2,66 \mathrm{GHz}$. De igual manera, en la Fig. 7 (b) se relacionan los resultados comparativos entre las medidas frente a las simulaciones del acoplamiento entre los puertos 1 y 2 de entrada. De la figura se nota que este parámetro posee un ancho de banda de $50 \mathrm{MHz}$ $(2,05 \%)$ para una frecuencia central $f_{0}=2,44 \mathrm{GHz}$.

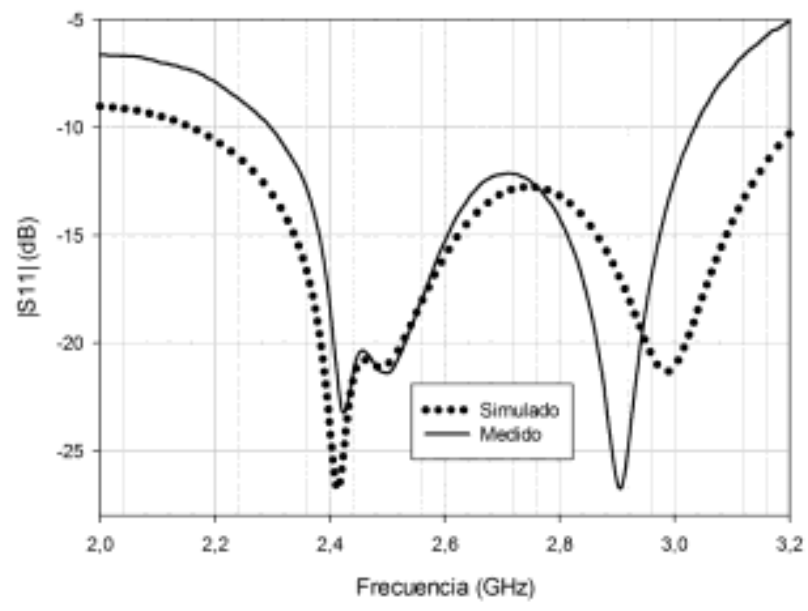

(a)

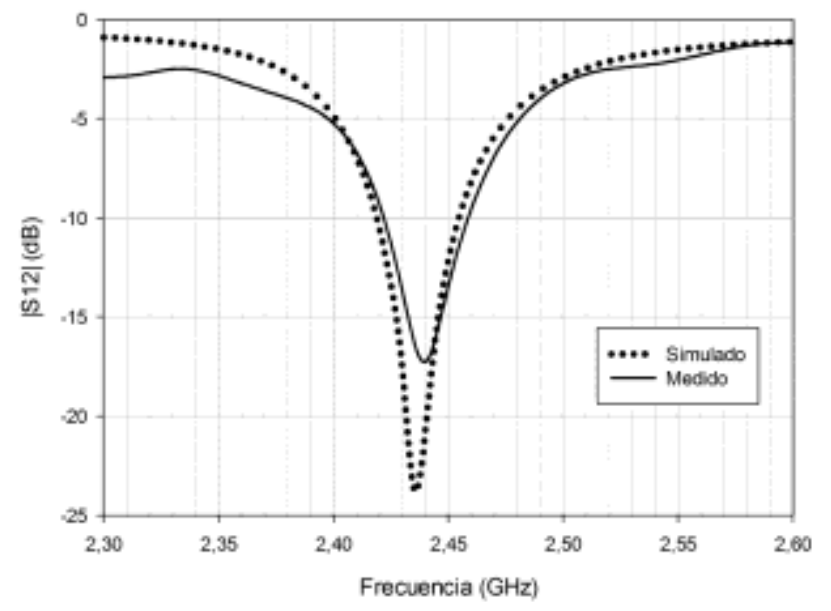

(b)

Fig. 7: (a) Medidas Vs simulaciones de parámetro $S_{11}$. (b) Medidas Vs simulaciones de parámetro de acoplamiento $S_{12}$.

En la Fig. 8 se aprecian los resultados de las medidas de ganancia llevadas a cabo en la cámara anecoica, frente a lo obtenido en las simulaciones. La ganancia máxima se presenta para una frecuencia central de $f_{0}=2,44 \mathrm{GHz}$ con un valor máximo de $G_{0}=6,04 \mathrm{dBi}$, la diferencia entre las dos curvas se explica por las pérdidas en los conectores, transiciones y cables utilizadas en el proceso de medida. Es de notar que en este tipo de antenas se logra reducir el nivel de la relación axial (a la frecuencia central) a valores inferiores de $2 \mathrm{~dB}$, en la medida en que el ancho de banda de impedancia $\left(S_{11}\right.$ y $\left.S_{22}\right)$ sea grande y se amplíe el ancho de banda de acoplamiento entre puertos $\left(S_{12}\right.$ y $\left.S_{21}\right)$; este último gracias a la presencia del acoplador. 


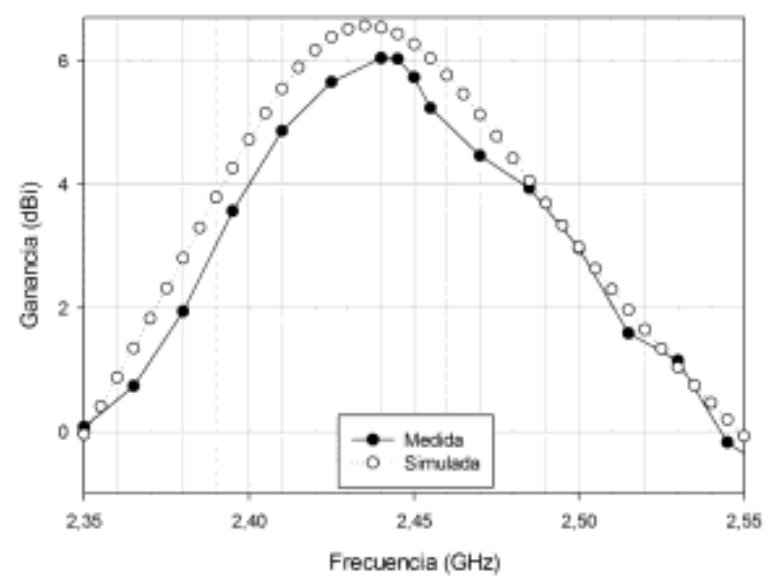

Fig.8: Ganancia medida versus simulada

En cuanto al comportamiento de la antena en diagrama de radiación, en la Fig. 9 se presentan los resultados de las medidas del diagrama en elevación de referencia (RHCP) y cruzado (LHCP) medidos a una frecuencia de $2,44 \mathrm{GHz}$, comparados con los resultados de las simulaciones. Como se observa, existen ligeras diferencias en los lóbulos posteriores entre el resultado de la medida y las simulaciones, los cuales son atribuidos a las limitaciones en la cámara anecoica disponible para tal fin, al igual que a los mecanismos de soporte tanto de las antenas de referencia como de medida. El diagrama de polarización cruzada muestra un factor de discriminación por polarización superior a $20 \mathrm{~dB}$ para el ángulo de máxima radiación. El ancho de haz de media potencia (HPBW) medido es de $79^{\circ}$ frente a $80^{\circ}$ obtenido en simulación. El ancho de banda de relación axial medido fue de $48.56 \mathrm{MHz}(2 \%)$ a una frecuencia de $2,44 \mathrm{GHz}$ para una referencia de $2 \mathrm{~dB}$, valor que resulta ser el doble del ancho de banda requerido.

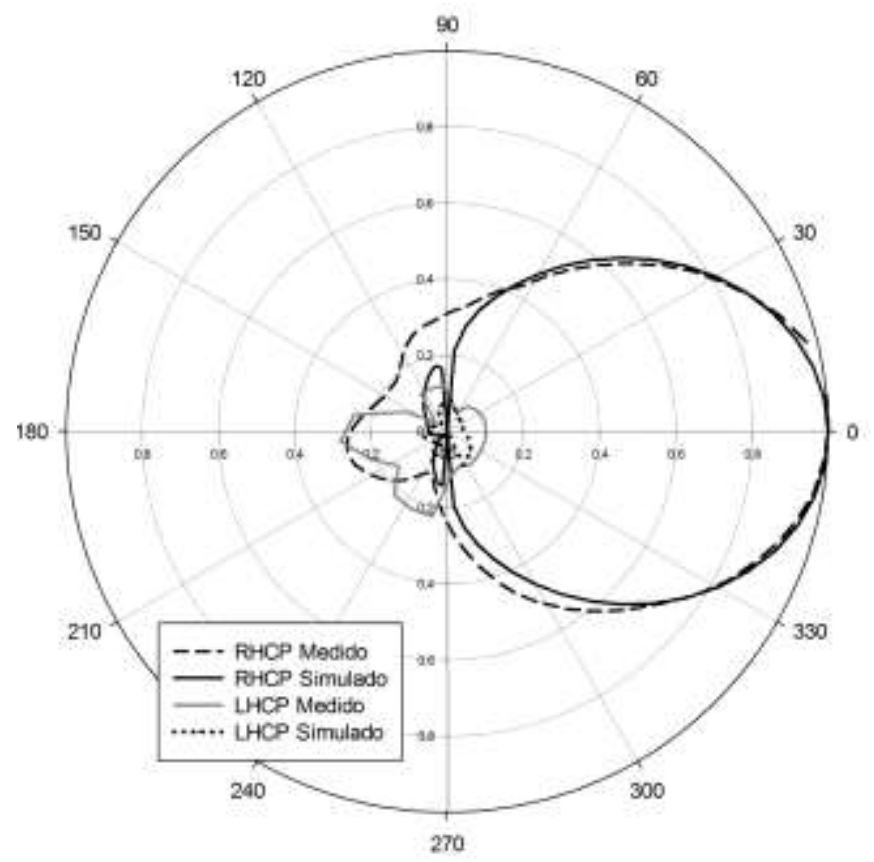

Fig. 9: Diagrama de radiación normalizado con corte en el plano XY

\section{CONCLUSIONES}

Este artículo presenta el diseño de una antena circular cortocircuitado con polarización circular y sentido de giro reconfigurable para aplicaciones en satélites CubeSat $1 \mathrm{U}$. El diseño propuesto logra una ganancia máxima de 6,04 dBi a la frecuencia central de 2,44 GHz. El ancho de banda de relación axial medido fue de $48.56 \mathrm{MHz}(2 \%)$ a la misma frecuencia y para una referencia de $2 \mathrm{~dB}$. La antena hace uso de un acoplador híbrido de $90^{\circ}$ que alimenta la antena en dos puntos, el cual permite de igual manera el cambio en el sentido de giro de RHCP a LHCP y viceversa. Así mismo, los resultados de las medidas del ancho de banda de impedancia son del 27,44\% para una magnitud del coeficiente de reflexión de $S_{11}<-10 \mathrm{~dB}$, en el rango de $2,295 \mathrm{GHz}$ a $3,025 \mathrm{GHz}$. Por último, los resultados de las medidas comparadas con las simulaciones son similares, sin embargo se aprecia una pequeña diferencia en las medidas del diagrama de radiación, debidos a las limitaciones de la cámara anecoica disponible para tal fin. 


\section{REFERENCIAS}

Balanis, C. A., Antenna Theory: Analysis and Design, 3a Ed., 844-846, J. Wiley, New York, USA (2005)

Berg, M., Ur, R., Lighari, R., Tuovinen, T., y E.T. Salonen, Circularly Polarized GPS Antenna for Simultaneous LHCP and RHCP Reception with High Isolation, Loughborough Antennas \& Propagation Conference (LAPC), 2-5, Loughborough, Reino Unido, 14 al 15 de Noviembre (2016)

Brookner, E., W.M. Hall, y R.H. Westlake, Faraday loss for L-band radar and communications systems, doi: 10.1109/TAES.1985.310634, IEEE Trans. on Aer. \& Elect. Syst., (en línea), 21(4), 459-469 (1985)

Cheng, Y., J. Fang, W. Lu, y H. Zhu, A Novel Reduce-Size Circularly Polarized Microstrip Antenna, $3^{\text {rd }}$ AsiaPacific Conference on Antennas and Propagation, 164-167, Harbin, China, 26 al 29 de Julio (2014)

Counselman, C.C., Multipath rejecting GPS antennas, doi: 10.1109/5.736343, Proceedings of the IEEE, (en línea), 87(1), 86-91 (1999)

Ernest, A., Y. Tawk, C.G. Christodoulou y J. Costantine, A deployable conical log-spiral antenna design for CubeSat applications. Proceedings of the 2012 IEEE International Symposium on Ant. and Prop., 1-2, Chicago, IL, USA, 8-14 de Julio (2012)

Gao, S., Luo, Q. y Zhu, F., Circularly Polarized antennas, 30-64, J. Wiley, New York, USA (2014)

Jackson, D.R., J.T. Williams, A.K. Bhattacharyya, R. L. Smith, S. J. Buchheit y S. A. Long, Microstrip patch designs that do not excite surface waves, doi: 10.1109/8.244643, IEEE Trans. Ant. \& Prop., (en línea), 41(8), 1026-1037 (1993)

Jie, A. M., M. F. Karim, L. Bin, F. Chin, y M. Ong, A Proximity-coupled Circularly Polarized Slotted- Circular Patch Antenna for RF Energy Harvesting Applications, IEEE Region 10 Conference (TENCON), 2027-2030, Marina Bay Sands, Singapur, 22 al 25 de Noviembre (2016)

Pozar, D.M y D.H. Shaubert, Microstrip Antennas, 105-148, IEEE Press, New York, USA (1995)

Pozar, D. M, Microwave Engineering, 3ª Ed., 333-337, J. Wiley, New York, USA (2005)

Rani, S., E.D. Singh, K. Sherdia, UWB Circular Microstrip Patch Antenna design simulation \& its Analysis, Int. Journal of Advanced Research in Comp. and Comm. Eng., 3(7), 7519- 7521 (2014)

Shackelford, A.K., Lee, K.F. y K.M. Luk, Design of small-size wide-bandwidth microstrip antennas, doi:10.1109/MAP.2003.1189652, IEEE Antennas and Prop. Magazine, (en línea), 45(1), 75-83 (2003)

Suárez-Fajardo, C. A., J. Carroll y G. A. Puerto, Antena Compacta para Aplicaciones en Satélites de Reducido Tamaño, doi: 10.4067/S0718-07642016000300018, Inf. Tecnol. (en línea), 27(3), 189-198 (2016a)

Suárez-Fajardo, C. A., D.R. Méndez, A. Pineda y G.A., Puerto Leguizamón, Antena de parche con sentido de giro reconfigurable para aplicaciones en satélites CubeSat, doi: 10.15446/dyna.v83n199.52449, DYNA, (en línea), 83(199), 157-164 (2016b)

Suárez-Fajardo, C.A., J.K. López y G. A. Puerto Leguizamón, Antena plana para aplicaciones en las bandas L1 y L2 de GPS, Ingeniería y Desarrollo, ISSN: 2145-9371, (en línea), 34(1), 248-265 (2016c), https://goo.gl/UcQVma (2016)

Wang, K. X., Li Gao y otros 5 autores, Multilayer wide-band patch antenna using L-probe and structure, $20124^{\text {th }}$ International High Speed Intelligent Communication Forum, 1-3, Jiangsu, China, 08 June (2012) 